\title{
The Correlation Between Blue Light Glasses and High School Student Retention in Class
}

\author{
Lauren P. Skelly ${ }^{1}$ and Patricia Talarczyk ${ }^{1}$ \\ ${ }^{1}$ Mentor High School, Mentor, OH, USA
}

\section{$\underline{\text { ABSTRACT }}$}

Blue light is known to have a multitude of negative effects on the health and well-being of someone who is frequently exposed to harmful light. Blue light glasses can provide temporary relief from blue light exposure by filtering out concentrations of blue light, discontinuing the transfer through the retina of the eye. The experiment consists of three reading comprehension tests, all of equal difficulty and length. There are two treatment groups: wearing blue light blocking glasses and not wearing blue light blocking glasses. After participating in the experiment, participants also answered a short survey regarding their experiences with blue light during school and outside of school. The results of the study were inconclusive, showing little to no correlation between wearing blue light blocking glasses and improved test scores. The survey allowed for further discovery about the negative implications resulting from blue light exposure, as many participants felt blue light has harmed their health and focus both in the classroom and outside of school. Since there was no statistical significance between treatment groups, considerations should be taken into perspective for future research to ensure more accurate results. Overall, blue light blocking glasses did not improve the ability to retain more information for high school student participants.

\section{The Correlation Between Blue Light Glasses and High School Student Retention in Class}

Blue light is known to cause a multitude of negative health effects such as headaches, eye strain, and lack of sleep. Blue light can be found through technological devices that display ecological lighting, a type of light pollution that can result in behavioral changes in organisms. This can be seen through different forms of light, as blue light is consistently found within the atmosphere derived from the sun's light rays. The light transfer is never-ending, as the sun is in constant exposure (Modi, 2019). Being consistently exposed to blue light, the effects this can have on one's health can cause a multitude of negative implications over an extended period (Vicente-Tejedor, 2018). The sleep patterns associated with REM sleep are commonly affected by blue light. This can cause disturbances throughout the night while blue light users sleep, in addition to difficulty falling asleep (Loredana-Sabina, 2019). Blue light suppresses melatonin production before sleeping, making it challenging for one to fall asleep without feeling tired, as well as difficulty staying asleep. The hormone melatonin is responsible for regulating sleeping cycles and waking up cycles. Additionally, short-term effects of blue light have been found recently in the current body of knowledge surrounding light exposure and increased electronic use (Tonetti, 2019). These effects pertain to headaches, as the muscles in the eyes must work harder to detect the wavelengths of the light as it is transformed from the eyes to the brain. The eye muscles needing to work harder can also lead to negative implications associated with eye strain and damage to the retina (Mastrota, 2019). The retina works to receive light and processes the light into neural signals, sending signals to the brain to interpret and process (Pauley, 2004). As a result, this can lead to eye strain since the eyes are working harder to deliver the light to the brain and process the light waves from the blue light. The effects of blue light can lead to difficulty sleeping, increased eye strain, and headaches, demonstrating the need for a solution to decrease light exposure. 
Understanding the negative implications associated with blue light, blue light glasses can provide a solution to ease side effects. Blue light glasses decrease the amount of blue light that is received by the eyes, by blocking out concentrations of the light (McEwen, 2015). When the light rays travel to the eyes, they are stopped by the glasses from continuing to transfer to the brain, as they absorb and refract the light rays. Similarly, without the eyes being able to transfer the waves into the brain for processing, short-term side effects associated with blue light are eliminated such as difficulty sleeping and focusing, headaches, and eye strain (Winx, 2018). The eyes will no longer need to be continuously exposed to stress by the blue light, not causing the retina excess strain when working to encode blue light within the brain. subsequently, sleep patterns can be improved, as the brain's melatonin is no longer affected by the blue light, decreasing the production and increased energy associated with the effects. This allows users to become tired when there is time to sleep, rather than having trouble sleeping (Lawrenson, 2017). In the same vein, this allows for the REM sleep patterns to be maintained, and consistent when sleeping throughout the night (Esaki 2016). As different concentrations of blue light can be filtered out by blue light glasses, users can control their exposure, catered to specific effects received from blue light, as well as how often they are exposed to the light. This allows blue light glasses to be a plausible solution for each user, as they are in control of their own experiences with the glasses. Blue light glasses demonstrate to be a solution for increased blue light exposure, decreasing negative implications such as eye strain, headaches, and difficulty sleeping.

By gaining an understanding of how blue light glasses can better those who commonly encounter blue light, can significantly improve the lives of countless users such as students who commonly are exposed to blue light while studying online. The purpose of blue light glasses can ease users' negative health implications, as well as improve retention. Through learning how blue light glasses can solve problems without harming health caused by blue light, can allow for exponential amounts of electronic use and sun exposure in the future. Moreover, blue light glasses enable increased screen time for those who need electronics in their daily lives. Users will be able to spend more time on electronics for work needs, school needs, or other tasks without fear of getting headaches or eye strain that will compromise the quality of work (Lievens, 2019). Understanding this, blue light glasses will be able to positively impact all users from each perspective, demonstrating the purpose of the research.

As blue light can affect countless people, there is a multitude of perspectives associated with blue light glasses. When placing a light on blue light users, consumers are often those who use electronic devices such as a computer or mobile phone on a daily scale. As of 2016, the United States population is composed of $89 \%$ that have at least a computer in their daily lives (Ryan, 2018). Students are common consumers due to the fact that many of their study and school resources have shifted online. In schools, many students spend class time on electronics as a source of in-class learning. Knowing students to be just one perspective blue light user, there are numerous varying perspectives associated with blue light. From an economic standpoint, the considered perspectives include the manufacturers of blue light glasses and the consumers of the glasses. The manufacturers of the glasses are able to make a profit through the consumers of blue light glasses, which allows the companies to improve the glasses for the consumers with increased profit. The consumers of blue light glasses such as students can thoroughly enjoy the use of electronic devices without receiving the negative implications relating to health risks with blue light exposure. As the manufacturers can make more profit from more purchases, this can also better the quality of the product to further decrease health risks through the medical lens. As the consumers of blue light glasses are given decreasing health implications such as headaches and eye strain, this can benefit the overall health and well-being of the users through the medical perspective, lessening how the light can be negatively perceived on health. From a scientific view, those who evaluate the effects of blue light, as well as blue light glasses have gained a more thorough understanding of how blue light can affect one's health, understanding that blue light is the primary cause of certain health detriments. Blue light glasses are able to positively affect the lives of those such as students who frequently are exposed to blue light through forms of electronic devices and as sun rays. Blue light glasses can better the health of users from multiple perspectives associated with blue light.

After evaluation of the current body of knowledge and perspectives regarding blue light and blue light glasses, a gap is demonstrated. As the research pertains to blue light users, a common health side effect to blue light 
exposure is sleep deprivation. As sleep deprivation from blue light can cause a lack of focus and retention of information, blue light does not always correlate with a lack of focus and retention. If one is not receiving enough sleep consistently, they may be unfocused and unmotivated to work (Adegbenro, 2019). The lack of sleep can also cause headaches for users, as their brains require more energy to process information efficiently. Since blue light causes a multitude of health implications, evaluating how blue light glasses affect the health of users may be predisposed (Lockley, 2007). While the effects may be due to the lack of sleep, the sleep deprivation is still a result of increased blue light exposure before sleep, so the effects are still directly correlated with increased blue light. Blue light glasses increase focus and retention of users, as negative health effects are associated with blue light. Understanding this, to what extent do blue light blocking glasses correspond with increased high school student retention in class?

\section{Method}

An experimental method design was used to collect data. This was composed of an experiment, in addition to a survey. An experiment was chosen to collect data because this allowed for larger sample size, as well as multiple treatment groups to be evaluated. The additional survey was chosen because this allowed for anonymous data to be collected from a wide range of participants to gather secondary data that could not be collected through the experiment portion of the method. This method differs from similar studies, as other studies focus solely on electronics in a school setting (Ajuwon, 2019), or focus on blue light glasses without a specific setting or task (Taylor, 2005). This method differs, as a combination of a school setting, reading comprehension, and evaluation of blue light blocking glasses are all incorporated to assess for a correlation. There were three different comprehension tests given to students who were separated into groups based on if they were assigned to the control group, the no glasses group, or the experimental group which was the group that wore the blue light blocking glasses. The blue light blocking glasses given to students were the Prospek-99 glasses from Spektrumglasses. These glasses had the lowest concentration of blue light concentration, blocking out 99\% of blue light (Spektrum Glasses). All participants were AP (Advanced Placement) Research class students. Each participant was randomly assigned to be in either the control or experimental group. After completing the comprehension analysis portion of the experiment, participants were then transitioned into a short survey evaluating their personal experiences and interactions with blue light exposure. This allowed for an in-depth understanding of how blue light affects high school students to add to the finding pertaining to the blue light effects on students (Sotty, 2013).

Participants were selected through the AP Research classes offered through the school. For this study, there were 58 total participants, all of whom were current AP Research students and members of the junior class. These AP Research students were selected as participants since the comprehension papers they needed to evaluate for the experiment portion were past AP Research papers. Current AP Research students would have the greatest understanding of the papers compared to other students without a research background, making AP Research students plausible participants. All participants are enrolled in a wide variety of other classes, being other Advanced Placement level courses, or regular or honors-level courses. Each participant gave assent to participate in the study, being involved in both the experiment and the survey. Since the experiment involved materials learned in the AP Research first semester curriculum such as the components to the AP Research paper rubric, participants were only those who are currently involved in the class. No students missed academic class time for their participation in the study and participation was completely optional. Participation took place in the classroom for each student, keeping the environment consistent with each participant, as well as the environment where the material learned took place. All information regarding each participant was kept anonymous, as well as their results from both the experiment and survey portion of this study. No student was aware of what the study was testing for previous to completing the study to ensure no bias was added to their results. In the assent form each student received, the requirements for the study, as well as any necessary information pertaining to their involvement in the study was clearly stated. Students were made aware through the assent form that some may be required to wear the blue light blocking glasses during the study if they are randomly 
selected to be in the experimental group portion of the study. All participation was completely voluntary for both the experiment and survey.

The data was collected over a period of a week-long process, in which each participant completed both the experiment and the survey on the same day. Not all participants completed the study on the same day. Participants were not aware as to whether or not they would be wearing blue light blocking glasses for the study until the day of their participation. During each school day, there are three different AP Research class times. During each class, students were selected in a predetermined, randomly generated order, to maintain organization and awareness of who was using the blue light blocking glasses during each class period. Two of the class periods were during the morning hours, while the third class period took place during the afternoon portion of the school day. Since there were 58 total participants, 29 students wore the blue light blocking glasses for the study, while the other 29 students did not wear the blue light blocking glasses. Each AP Research class has the same teacher, who allowed the students to participate during class time as needed. Students could opt to participate during a different day if they felt they could not participate on their assigned day due to time restraints and other personal commitments.

For the first part of the study, participants needed to complete the experiment portion. The experiment consisted of three Google Forms quizzes, each with 10 multiple-choice questions. Each participant was assigned one of the three AP Research paper comprehension quizzes and was to read the paper and take the quiz correlating with what paper they read. There were three different papers to read with corresponding Google Forms to ensure participants would not share details of the quiz with those who were participating at a later date or time. In each quiz, the 10 questions were created in a manner that allowed them to be of equal length and difficulty to ensure no bias and accurate results. In each quiz, the questions were created from each section of the papers; there was one question from the abstract section, two from the introduction, one from the method, one from results, two from analysis, and three from the conclusion. Students were to read each component of the paper, ensuring they were able to answer each question all the way through the content in the conclusion. Before beginning to read their assigned papers, students who were assigned to wear the blue light blocking glasses were given the glasses and instructed to put them on. Regardless of the treatment group students were in, all participants were given the same instructions: turn the brightness on their school-issued MacBook to the maximum brightness setting (Twinning, 2002). Students were then allowed to begin reading their assigned papers. Students were instructed to not refer back to the paper while completing the quiz, as well as keeping their brightness setting on maximum. Participation in this study took the duration of the class period for most students. Understanding this, since there were only three pairs of blocking glasses, students assigned to this group had to go three at a time per class period. On the other hand, participants in the group without the blue light blocking glasses could go at any time on whichever preferred day since they did not have to rotate with the glasses. The names of students and who was assigned which group was written down on the three-class lists and their names were checked off after their participation to ensure each student who assented to participate had a chance to do so. This also allowed for the attendance of who was to read each paper and take the assigned quiz. After completing the quiz, students were then directed to take a survey.

The survey was given to students in the same Google Form the comprehension quiz was entered in. Since there were three Google Forms quizzes for each paper, each Google Form had the same survey. The full survey and questions can be viewed through Appendix A. At the beginning of the survey, a short description of what blue light is, as well as where blue light is found and the effects it can have on health was mentioned. This allowed participants to understand the impacts of blue light and to answer the survey questions most efficiently. The survey was composed of questions asking students to share their experiences with blue light, both in school, as well as in their day-to-day lives. Since this study is evaluating the effects of blue light on student retention in class, the survey evaluated if students themselves feel as though blue light use is affecting their education, in addition to their ability to focus and retain information in class. They were asked how much time they spend on their MacBook during the school day and how much time they spend on their MacBook after the school day. Students were also asked how they feel their blue light exposure affects their health in terms of headaches, eye strain or pain, ability to sleep, and other health implications associated with blue light. This allowed for a comprehensive understanding of how blue light affects the students 
in school and their daily lives. Since the amount of time participants spend a day exposed to blue light was also asked, this allowed for an evaluation of exposure time and the correlation this had with health implications. With the survey involving questions about personal experiences and feelings, participation was voluntary, and participants were required to assent previous to beginning the survey and test. The survey allowed for an understanding of how blue light affects student health, as well as how they feel it impacts their education.

\section{Results}

After gathering data from the method, the results from each treatment group show the group with the blue light blocking glasses performed only slightly higher than the non-blue light blocking glasses group. Since each treatment group was given the same three tests, there was no influence on the scores of participants from an outside perspective. The average test score out of the 10 questions for the glasses group was 6.346, while the non-glasses group scored an average of 6.269 out of 10 . The glasses scored only $0.077 \%$ higher than the non-glasses, demonstrating little to no correlation. In both treatment groups, however, the scores for participants who read paper two and took the correlating quiz for paper two scored higher. Since this remained true for each treatment group, this demonstrates that wearing or not wearing blue light blocking glasses was not the cause for this score increase. Each average test score per test for wearing blue light blocking glasses can be seen in Figure 1. The average test score per paper for the not wearing blue light blocking glasses group can be seen in Figure 2. These results show that when the study was implemented, students performed just as they would in a natural setting without the blue light glasses. The blue light glasses did not harm the scores of participants, yet had little to no increase in the scores. The lack of statistical significance demonstrates that the performance of students is not affected by blue light blocking glasses.

Figure 1. Average Score With Blue Light Glasses

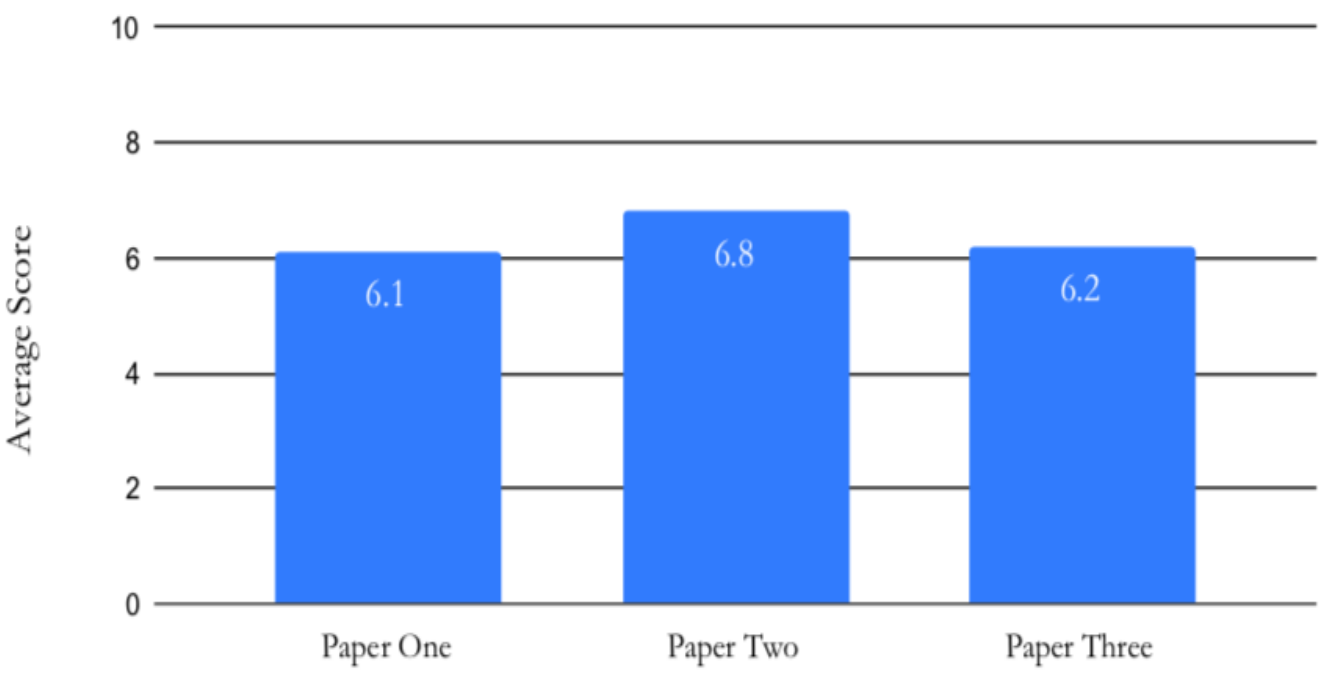

AP Research Sample Paper 


\section{Figure 2. $\quad$ Average Score Without Blue Light Glasses}

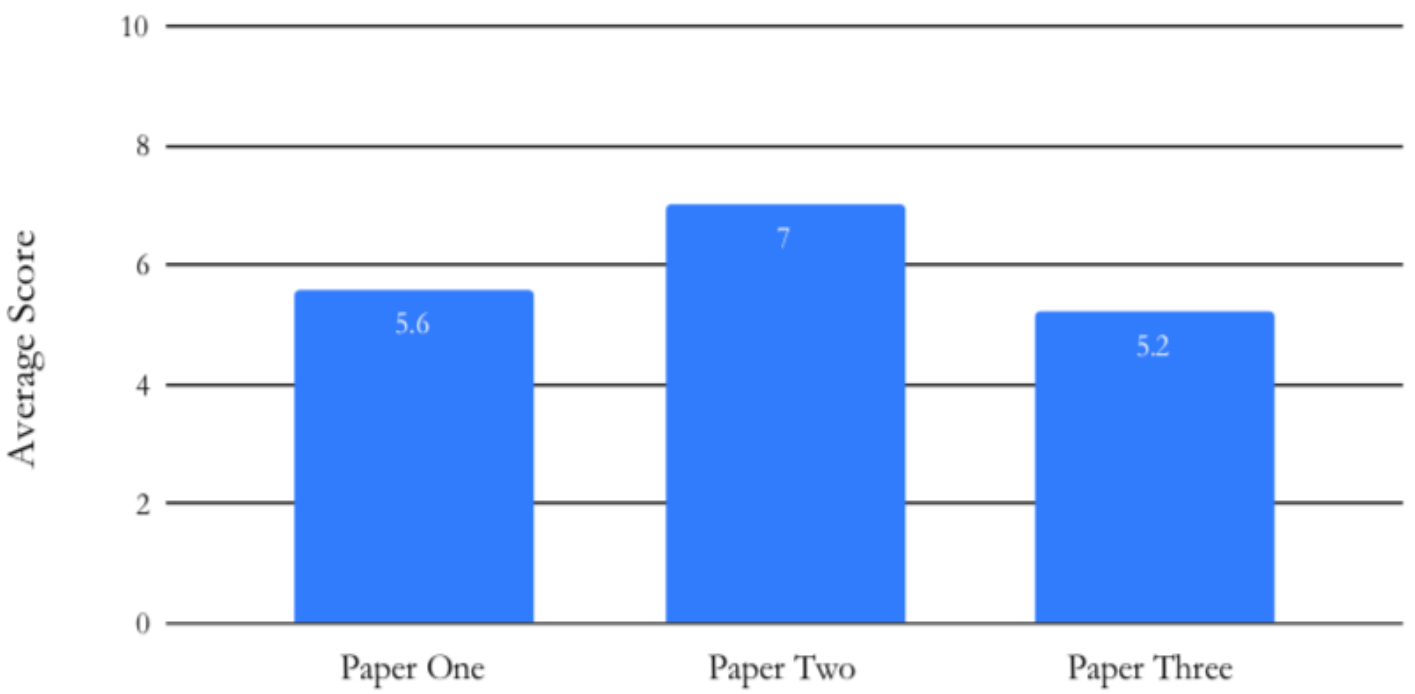

AP Research Sample Paper

After an evaluation of the surveys taken by each participant, results are evidence that a multitude of high school students experiences negative health implications as a result of increased blue light interaction. Most students stated they spend between five and six hours a day on their school-issued MacBooks. With this amount of time, 52\% of students stated that they feel blue light affects them negatively both inside and outside of school. Many stated they receive headaches or eye strain as a result of increased time on their MacBooks, which causes difficulty studying and focusing on tasks online. Students also felt that excessive amounts of blue light exposure contributed to difficulty sleeping, which is also a determining factor of lower student test scores. Additionally, the data also concluded that students felt better while wearing blue light blocking glasses, having fewer headaches, eyestrain, and fatigue. A multitude of students felt that wearing the blue light glasses increased their attentiveness due to the lack of health problems associated with the blue light that had been discontinued by the glasses. This shows that while the glasses did not improve test scores significantly, they were effective in improving test conditions and confidence while taking tests. Through implementing the survey, awareness about the effects of blue light on students is evident. This allowed students to actively evaluate their stance on blue light to pursue change as needed in their daily lives. Understanding these results, it is conclusive that increased amounts of blue light cause detriments to the health of high school students. 


\section{Figure 3. The Harms of Blue Light on Student Health}

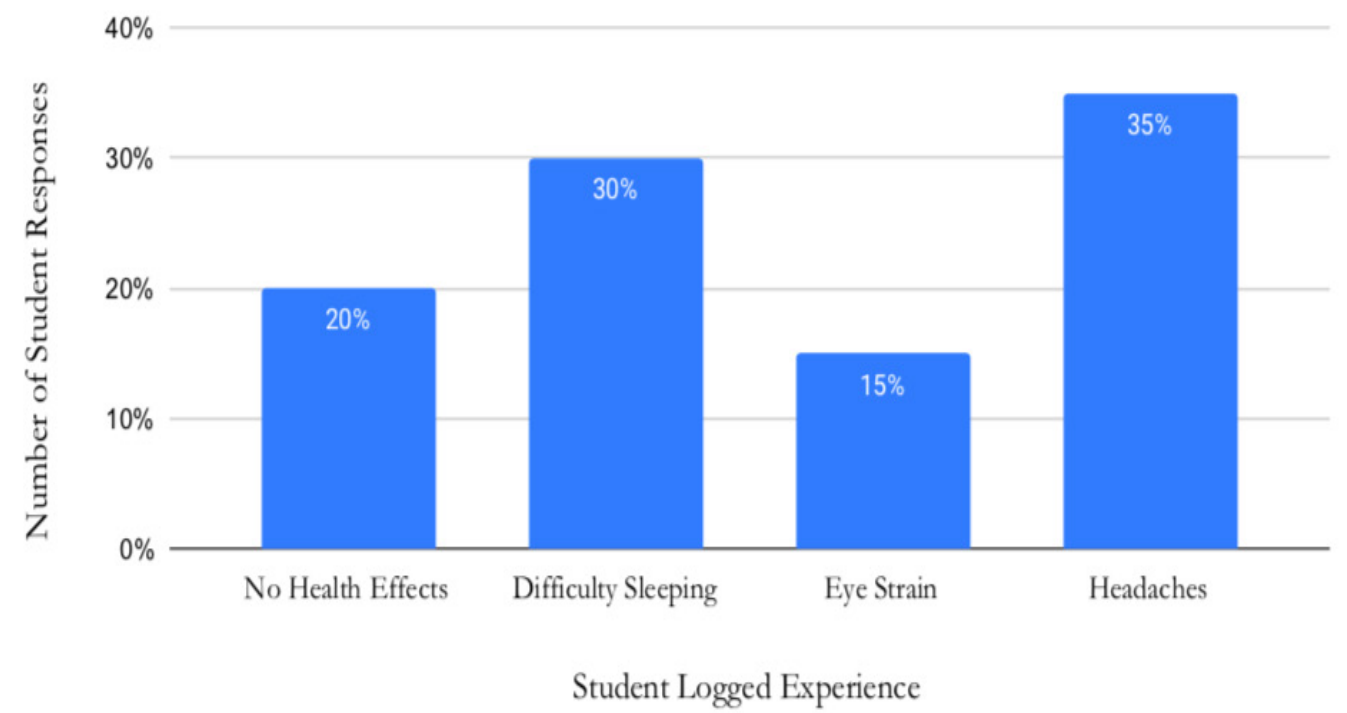

\section{Analysis}

Through evaluating the results, a new understanding has been met. Knowing this, the initial hypothesis that the blue light blocking glasses would improve student retention and focus is incorrect. Each treatment group's scores were close in range, demonstrating no significant gap in scores as a result of the blue light blocking glasses. A Spearman's Rho statistical test was used, as this type of statistical test is used to measure the strength between multiple variables; these variables are the two treatment groups being wearing blocking glasses and not wearing blocking glasses (Quick Statistics Calculators, 2019). This statistical test was used to determine the p-value of the data, which amounted to the value of 0.46168 . This p-value was not statistically significant, which further demonstrates the lack of correlation between the treatment groups of participants wearing or not wearing the blue light blocking glasses. This shows that the blue light blocking glasses are not useful at improving retention for high school students on their MacBooks. Despite the lack of improved scores, the blue light glasses are known to decrease health problems associated with blue light and were successful at this according to the survey results. Understanding this, while the blue light glasses were unsuccessful at improving test scores, they were successful at improving conditions for test-taking for each participant. This shows that even though the glasses are not directly correlated with test scores, the improved health conditions could have prevented participants from getting worse test scores, had the test been given over a longer time. This limitation can account for the lack of statistical significance between each treatment group, as the participants might not have been exposed to blue light for a significant enough time for the blocking glasses to improve test scores.

In addition, numerous other limitations could have impacted the study. The lack of time participants had to complete the study could have played a role, as some students could have felt rushed to complete the study. Since the study was conducted during a class period, the time to read the paper and answer the corresponding quiz was around 45 to 50 minutes. While this is a great length of time, the papers participants had to read for the study were lengthy. Some participants may read slower than others, leading to a sense of rush to complete the study. In conjunction, the scope of participants was limited to one selected local high school. Neighboring schools do not have the technology necessary to complete the study. All students are granted access to their MacBook during their four years at the high school, so this school was the only source of participants. If more local schools were involved, a deeper in-depth evaluation could have been done through a more diverse pool of students with differing educational backgrounds. 
Additionally, the knowledge gap associated with decreased sleep in frequent blue light users is a limitation. Any participants could have been sleep-deprived more than other participants, which could have affected their performance in the study. Any confounding variables not accounted for in the study could also limit the results of the study. These limitations played a key role in the study, potentially impacting the results of the study.

Understanding that the blue light glasses were unsuccessful at improving test scores but were successful at improving conditions for test-taking, this can expand upon the known body of knowledge of blue light and the effects. This study further demonstrated the multitude of negatives blue light possesses on the health of people who are exposed consistently, as participants answered in the survey the ways blue light affects their health poorly. Since the follow-up survey questions demonstrated the success of blue light glasses in terms of decreasing negative blue light effects, this expands on the known information in the current knowledge base. This supports the notion that blue light glasses are successful at filtering out high concentrations of blue light exposure, hence reducing negative health repercussions associated with the harmful light. These results further answer the question of the success of blue light glasses for their intended purpose of filtering out blue light. Also, the results add to the current knowledge gap of how this relates to students and working online such as reading. The understanding was reached from the high school student participants about how the blue light glasses improved their quality of studying online, leading to the conclusion that blue light glasses can improve the quality of studying and working online. Despite the quality of working online improving as a result of the glasses, the lack of increase in scores shown previously in Figure 1 and Figure 2 assists in demonstrating that blue light glasses solely improve the quality of working online, rather than the work itself, such as the test scores from reading comprehension that was evaluated.

\section{Conclusion}

The blue light glasses were slightly successful in improving the retention of students while reading and studying. Despite this, the scores were not significant in determining that the blue light blocking glasses were able to improve the retention of information for high school students. With these results, the implications of the study allow for a better understanding of potential future directions for research. Future research can redirect the focus of this study away from solely high school student participants to participants from all grade levels, as well as college participants. This will allow for a more comprehensive understanding of what audience is most affected by the use of blue light blocking glasses. Additionally, future research can specify what area of studies could be most beneficial in terms of using blue light glasses. This study focused on reading comprehension, but future studies can focus on other areas of studies. Future studies can also evaluate the results of this study on other electronics students commonly used for studying, such as iPads and Laptops for example. There are countless directions for future research to better understand blue light and all corresponding impacts.

Implications of the lack of differences in scores can allow for future researchers to make adjustments to the research process as well. For a follow-up study, introducing participants to the blue light blocking glasses previous to participation in the study might create a calmer, more relaxed environment, leading to better test scores. This will ensure participants will already be aware of the discoloration on their MacBook screen that the glasses cause due to the tinted orange color of the glasses, as well as how the glasses feel on their face. Lack of familiarity and awareness could have been a contributing factor to the results of the study, influencing the performance of the treatment groups. Connecting to awareness, in future research all participants should also be separated by treatment group, as well as by the assigned test they were given. Participants who did not wear the blue light blocking glasses could have been less focused while taking the assessment, due to curiosity about the glasses their peers were wearing. This would lead to a lack of focus, which can decrease the amount of retained information from the comprehensive test. Separating treatment groups would eliminate this implication and allow full focus on the assignment. Since there was no statistical significance between the treatment groups and participant performance, future research should include necessary changes to ensure more accurate results. This study was able to assess the correlation between wearing blue light 
blocking glasses and changes in terms of reading comprehension, finding little to no statistical significance between the two. With necessary changes for future research, new findings can demonstrate a potentially stronger correlation between variables and further a more in-depth knowledge base on blue light glasses and their potential in terms of academic success.

\section{Acknowledgments}

I would like to thank my advisor Mrs. Patricia Talarczyk for her support with this project.

\section{References}

Adegbenro, J. B., \& Olugbara, O. O. (2019). Investigating Computer Application Technology Teachers' Procedural Knowledge and Pedagogical Practices in ICT- Enhanced Classrooms. Africa Education Review, 16(1), 118. https://doi.org/10.1080/18146627.2017.139451

Ajuwon, G. A., \& Ajuwon, A. J. (2019). Teaching high school students to use online consumer health resources on mobile phones: outcome of a pilot project in Oyo State, Nigeria. Journal of the Medical Library Association, 107(2), 194-202.

Lawrenson, J. G., Hull, C. C., \& Downie, L. E. (2017). The effect of blue-light blocking spectacle lenses on visual performance, macular health and the sleep-wake cycle: a systematic review of the literature. Ophthalmic \& Physiological Optics, 37(6), 644-654. https://doi.org/10.1111/opo.12406

Lievens, C. (2019). Blue light: Why it matters: As digital device usage increases, so does potential for ocular damage. Optometry Times, 11(3), 28-29. Retrieved from http://search.ebscohost.com/login.aspx?direct=true $\& \mathrm{db}=$ asn $\& A N=135522577 \&$ site $=$ ehost-live

Lockley, S. W. (2007). Safety considerations for the use of blue-light blocking glasses in shift-workers. Journal of Pineal Research, 42(2), 210-211. https://doi.org/10.1111/j.1600-079X.2006.00402.x

Loredana-Sabina, P., Perri, D., Vlad, B. A., Ciubara, A., Marilena, M., \& Virginia, M. (2019). The Effects of Blue Light in Modern Society. BRAIN: Broad Research in Artificial Intelligence \& Neuroscience, 10, 5-11. Re-

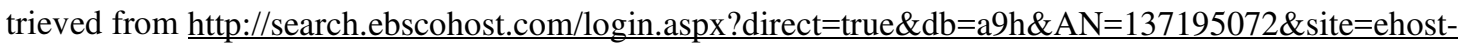
$\underline{\text { live }}$

Mastrota, K. M. (2019). How to manage digital eye strain: Educate patients on damaging vision effects linked to regular digital device use. Optometry Times, 11(1), 8. Retrieved from http://search.ebscohost.com/login.aspx? direct=true $\& \mathrm{db}=$ asn $\& A N=133965238 \&$ site $=$ ehost-live

McEwen, R. N., \& Dubé, A. K. (2015). Engaging or Distracting: Children's Tablet Computer Use in Education. Journal of Educational Technology \& Society, 18(4), 9-23. Retrieved from http://search.ebscohost.com/login.aspx?direct=true $\& \mathrm{db}=$ asn $\& A N=110247500 \&$ site $=$ ehost-live

Pauley, Stephen M.( Mar. 2004). "Lighting for the Human Circadian Clock: Recent Research Indicates That Lighting Has Become a Public Health Issue." Medical Hypotheses, intl.elsevierhealth.com/journals/mehy. 
Pratibha Modi, Kamlesh Jha, Yogesh Kumar, Tribhuwan Kumar, Ramji Singh, Abhilasha Mishra. (2019). The effect of short-term exposure to red and blue light on the autonomic tone of the individuals with newly diagnosed essential hypertension. Journal of Family Medicine \& Primary Care, 8(1), 14-21. https://doi.org/10.4103/jfmpc.jfmpc 37518

Quick Statistics Calculators. 2019. Which Statistics Test Should I Use? www.socscistatistics.com/tests/what stats test wizard.aspx.

Ryan, Camille. (2018). "Computer and Internet Use in the United States: 2016." The United States Census Bureau, www.census.gov/library/publications/2018/acs/acs-39.html.

Sotty, C. (2013). The Benefits and Dangers OF BLUE LIGHT. Review of Optometry, 150(7), 39. Retrieved from http://search.ebscohost.com/login.aspx?direct=true \&db=a9h\&AN=89413199\&site=ehost-live

Spektrum Glasses. “Computer Glasses: Best Blue Light Blocking Glasses.” www.spektrumglasses.com/.

Taylor, R.H., and M.J. Mortimer. (2005). "The Use of Tinted Glasses in Childhood Migraine.” American Headache Society, John Wiley \& Sons, Ltd, headachejournal.onlinelibrary.wiley.com/doi/abs/10.1111/j.15264610.1991.hed3108533.x.

Tonetti, L., \& Natale, V. (2019). Effects of a single short exposure to blue light on cognitive performance. Chronobiology International: The Journal of Biological \& Medical Rhythm Research, 36(5), 725-732. https://doi.org/10.1080/07420528.2019.1593191

Twining, P. (2002). Conceptualising Computer Use in Education: introducing the Computer Practice Framework (CPF). British Educational Research Journal, 28(1), 95-110. https://doi.org/10.1080/01411920120109775

Vicente-Tejedor, J., Marchena, M., Ramírez, L., García-Ayuso, D., Gómez-Vicente, V., Sánchez-Ramos, C., Germain, F. (2018). Removal of the blue component of light significantly decreases retinal damage after high intensity

Winx, B. (2018, September 4). Blue Light Glasses Help You Focus On Better Sleep. Retrieved from https://bluwinx.com/sleep/blue-light-glasses-better-sleep-back-to-school/

Yuichi Esaki, Tsuyoshi Kitajima, Yasuhiro Ito, Shigefumi Koike, Yasumi Nakao, Akiko Tsuchiya, Marina Hirose \& Nakao Iwata (2016) Wearing blue light-blocking glasses in the evening advances circadian rhythms in the patients with delayed sleep phase disorder: An open-label trial, Chronobiology International, 33:8, 10371044, DOI: $10.1080 / 07420528.2016 .1194289$ 